\title{
Towards Building Asset Registry in Emergency Response
}

\author{
Shahram Mohrehkesh, Aaron Walden, Xianping Wang, Michele C. Weigle and Stephan Olariu \\ Department of Computer Science, Old Dominion University \\ Norfolk, VA USA 23529-0162 \\ Email: \{smohrehk,awalden,wxianpin,mweigle,olariu\}@cs.odu.edu
}

\begin{abstract}
This work proposes an algorithm for asset registry in emergency situations. Specifically, we take the view that first responders need to harvest the unknown capabilities of sensors in an area affected by an emergency. We show how a first responder can estimate the number of sensors with a desired sensing capability in an efficient fashion. At the heart of our algorithm is a simple probabilistic counting strategy. Our theoretical predictions were confirmed both by simulation and by implementation on IRIS sensor motes. Our theoretical and experimental results show that the error percentage of the capability collection is very low.
\end{abstract}

\section{INTRODUCTION AND MOTIVATION}

In an emergency, obtaining relevant information on which first responders (FRs) can act is a challenge. In particular, FRs must have knowledge of the assets available in order to tailor an effective emergency response strategy [1]-[3]. The main contribution of this paper is to address the task of asset registry when the resources of independently-deployed sensor networks need to be harnessed to help FRs.

We are interested in building, in an opportunistic fashion, a registry of the sensing capabilities of devices (mostly sensors) present at the scene of the emergency. Our vision is that while these devices may have been deployed over time by various authorities in support of missions unrelated to the emergency, their corporate capabilities could, and should, be made available to emergency personnel.

Imagine the following scenario. A building is on fire and several injured people need to be rescued. First responders arrive at the scene to rescue the injured and to put out the fire. While the FRs may already know some information such as the building topology, there is other situational information (e.g., fire location, location of injured people, practicable access routes) unknown to them and yet crucial to devising a successful rescue strategy [2].

Further imagine that a FR has been tasked with charting a safe path for evacuating the wounded. The FR must first assess the feasibility of several possible evacuation routes in terms of maximum temperature and smoke density. To evaluate the routes, the FR will enlist the cooperation of temperature sensors and smoke detectors by first finding the number and locations of the sensors and detectors in the relevant areas.

It is not possible to implement a complex protocol in emergency situations to collect the information about sensing capabilities of sensors because time is critical and the value of

\footnotetext{
Work supported by NSF grant CNS-1116238
}

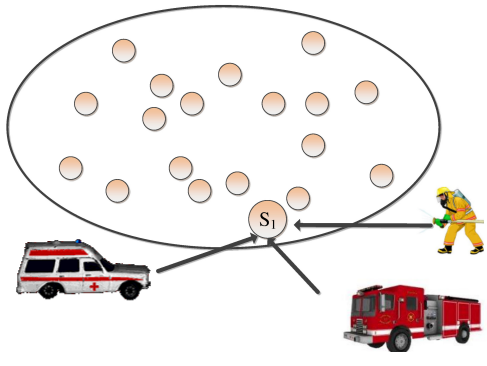

Fig. 1. Localization in emergency response.

information degrades as time passes [4], [5]. Therefore, a simple and light-weight protocol is required. Since an approximate asset registry will suffice for the purpose of deploying sensors in emergency situations, we take a probabilistic counting approach, which results in a light-weight and efficient protocol.

In the remainder of this paper, we first describe our assumptions and required infrastructure for our asset registry system in Section II. In Section III, we discuss our probabilistic counting strategy. In Section IV, we present a validation of our asset registry through simulation and implementation in IRIS motes. Finally, Section V offers concluding remarks.

\section{ASSUMPTIONS AND INFRASTRUCTURE}

\section{A. Localization}

Since most sensors are not equipped with GPS and the performance of GPS in indoor scenarios is poor, a new localization method is required to determine sensor nodes' locations relative to other sensor nodes and FRs. We propose using the GPS of other systems present at the emergency area to find the relative locations of sensor nodes. Each FR communication device, ambulance, or fire-truck is equipped with a GPS system. Since they do not have power limitations and they are operating outdoors, their GPS will be able to determine their exact location. These devices will start communicating with the node closest to them. The node, e.g. $S_{1}$ in Figure 1, will determine its location with triangulation methods based on the location received from the GPS-equipped devices and its estimated distance relative to them.

After only three adjacent sensor nodes know their locations, an indoor localization method, e.g., [6], could be used to specify the location of all nodes in the network. After sensor nodes know their position relative to one another, FRs can run any operation that needs location information. For example, one FR may need to find a path to an injured person. 


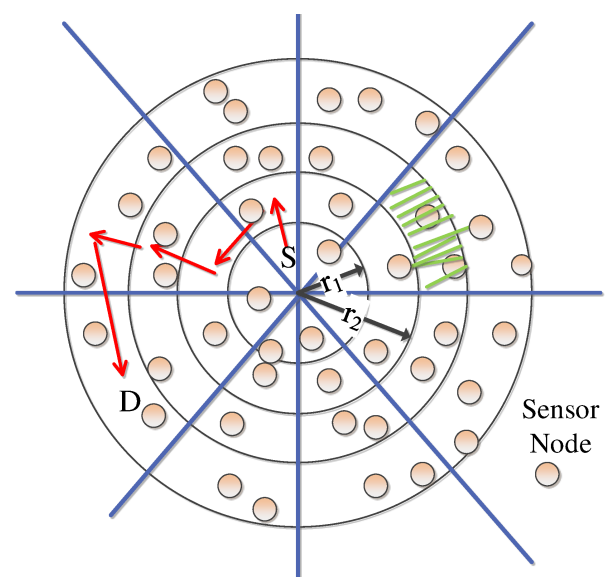

Fig. 2. Illustrating the VI centered at a FR.

\section{B. The virtual infrastructure}

In addition to localization, to support these tasks, a logical structure of nodes that a FR could interact with is required. For this purpose, the FR establishes a virtual infrastructure (VI), similar to the one proposed by Olariu et al. [7], [8], in a disk of a suitable radius centered at him or herself. The VI affords a coarse-grain sensor localization by mimicking a discretized polar coordinate system centered at the FR, as illustrated in Figure 2. The disk around the FR is partitioned into concentric circles, referred to as coronas and equiangular wedges. The intersection of two adjacent coronas and wedges determine a sector.

All the sensors in a sector acquire the same ID, which is the tuple consisting of the corresponding corona and wedge number. For example, the dashed area in Figure 2 is the sector with corona ID 3 and wedge ID 1, where coronas are numbered from 1 to 4 from the inner corona to the outer corona, and wedges are numbered 1 to 8 in a counter-clockwise direction.

The radii can be designed so that all sectors (resp. coronas) have the same area. More specifically, assume that the FR decided to partition the area of interest into $m$ coronas. Assuming that the radius of the innermost corona is $r_{1}$, then elementary calculations reveal that in order for all the coronas to have the same area, it suffices that for all $i,(1 \leq i \leq m)$

$$
r_{i}=\sqrt{i} \cdot r_{1} .
$$

The number of coronas and wedges in the VI is dependent on both the application and sensor density. For applications which require a finer location granularity, the number of coronas and wedges must be larger. But, very small sectors may create connectivity problems between sectors, which hinder the flow of information. In addition, a large number of sectors introduces overhead, i.e., delay in collecting the information or communication with nodes in different sectors.

The optimum number of sectors can be formulated as follows. The area of interest could be divided into $s$ sectors consisting of $j$ wedges and $k$ coronas, i.e., $s=j \cdot k$. Assume that $R_{\text {long }}$ is the maximum communication range for the FR.
Thus, we wish to maximize $s=j \cdot k$ subject to

$$
\sqrt{k} \cdot r_{1} \leq R_{\text {long }}, \quad S_{\min } \leq \frac{\pi \cdot r_{1}^{2}}{j} \leq S_{\max },
$$

where $S_{\min }$ and $S_{\max }$ are the minimum and maximum sector size, respectively. These are application-dependent.

This problem formulation maximizes the number of sectors $s$ while limiting it by the defined constraints. The first constraint limits the maximum radius to the maximum communication range of the FR. The second constraint provides a lower limit on sector size. For example, in an area with $300 \mathrm{~m}$ radius, sectors of $1 \mathrm{~m}^{2}$ are not required, and $S_{\min }$ can be set to higher values, e.g., $100 \mathrm{~m}^{2}$. Likewise, $S_{\max }$ can be selected based on the communication range of sensors, which is shorter than that of the FR. For example, to have communication between sectors in low density scenarios, $S_{\max }$ can be set to be slightly smaller than the communication range of sensors. The above optimization problem is a combinatorial optimization problem since $k$ and $j$ can only have discrete values.

The VI supports the deployment of many mechanisms and protocols. For example, a clustering mechanism could be considered, where nodes in one sector make up a cluster. We use the VI to set up a registry of the capabilities of the sensor nodes. This information is required for better scheduling and assigning of tasks to sensors to support the mission of FRs. Finally, the VI enables the deployment of efficient information collection methods that are based on the location of nodes. For example, redundant information collection from nodes in the same sector can be avoided since data of nodes with the same relative location would likely be similar.

As an example scenario, a FR could exploit the VI for the purpose of identifying evacuation routes from a burning building. For example, if one FR is at sector $S$ (Figure 2) and needs to move to sector $D$ to rescue an injured person, the VI will support its mission by specifying a path, determined through cooperation of sensor nodes. These requests are dynamically defined by the FRs as performing different duties to finish the mission. For example, after the FR finds the injured person, he needs to find a safe path to take the person to safety.

\section{Reprograming the motes}

The last assumption required for asset registry is the ability to reprogram sensor nodes. From a technical viewpoint, the ability to reprogram sensor nodes, such as Mica-Z and IRIS motes, exists through systems such as Deluge [9]. In terms of authorization to retask, we consider two possibilities: (I) the owner of sensor network would grant the FRs access to reprogram the sensors, e.g. through a special password; or (II) there are some built-in capabilities in the sensors that allow retasking them in emergency situations. It would be similar to the capability of emergency calls [10] on cell phones that operate on the cellular network of any provider.

\section{The COUNTING PROCESS}

The goal of this section is to define how the number of sensors and their capabilities can be collected in a probabilistic fashion. We first introduce a probabilistic counting process 
which is used to collect the number of sensors. Then, we show how this can be extended for high density networks to keep the accuracy of the method scalable.

\section{A. Our probabilistic counting process}

In the early stages of mission planning, a FR wants to obtain a good estimate of the number of sensors with a desired capability in an area of interest. For definiteness, assume that there are $N$ such sensors. Having selected a suitable positive integer $n$, the FR instructs each sensor with the desired capability to generate a bit vector of size $n$ initialized to all os and to set, with probability $\frac{1}{n}$, a random bit therein. Assuming an ideal communication channel (no interference, and no collision caused by simultaneous transmissions), the bit vectors of the various sensors are aggregated by OR-ing them together following the procedure described in [11].

The number of $1 \mathrm{~s}$ in the aggregated bit vector represents an estimate of the number $N$ of sensors that have the desired capability. However, since the sensors select their bit vector independently, there is a chance that two or more sensors will set the same bit. Therefore, we are interested in calculating the expected number of $1 \mathrm{~s}$ in the aggregated bit vector.

The fact that each sensor sets a bit with probability $\frac{1}{n}$ is tantamount to the sensor placing, uniformly at random, a ball in one of $n$ bins. By virtue of OR-ing the individual bit vectors together, each sensor's selected bit is guaranteed to be reported in the aggregated bit vector. Thus, we can model the problem as an instance of the well-known Maxwell-Boltzmann statistics [12] and that all the $n^{N}$ distinct possibilities are equiprobable.

Of interest is the expected number of occupied bins or, equivalently, the number of $1 s$ in the aggregated bit vector. With the $i$-th bit in the bit vector we associate an indicator random variable $X_{i}$ defined as follows

$$
X_{i}= \begin{cases}1 & \text { if bit } i \text { is set } \\ 0 & \text { otherwise }\end{cases}
$$

In other words the number $Y$ of $1 \mathrm{~s}$ in the aggregated bit vector is $Y=X_{1}+X_{2}+\cdots+X_{n}$. It is easy to see that

$$
\operatorname{Pr}\left[\left\{X_{i}=1\right\}\right]=1-\left(1-\frac{1}{n}\right)^{N} .
$$

Using the linearity of expectation, we write

$$
\begin{aligned}
E[Y] & =\sum_{i=1}^{n} E\left[X_{i}\right] \\
& =\sum_{i=1}^{n} \operatorname{Pr}\left[\left\{X_{i}=1\right\}\right] \\
& =\sum_{i=1}^{n}\left[1-\left(1-\frac{1}{n}\right)^{N}\right] \\
& =n\left[1-\left(1-\frac{1}{n}\right)^{N}\right]
\end{aligned}
$$

To avoid the computation in (2), we can use the limit

$$
\lim _{n \rightarrow \infty}\left(1-\frac{1}{n}\right)^{n}=e^{-1}
$$

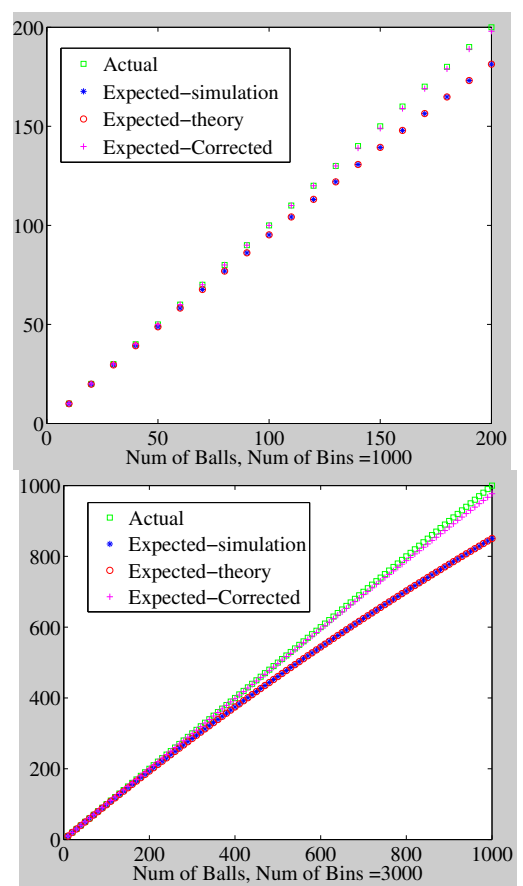

Fig. 3. Comparison of probabilistic counting and actual number of balls (Top - 1000 bins, bottom - 3000 bins).

to approximate $E[Y]$ as follows

$$
\begin{aligned}
E[Y] & =n\left[1-\left(1-\frac{1}{n}\right)^{N}\right] \\
& =n\left[1-\left(1-\frac{1}{n}\right)^{n \frac{N}{n}}\right] \\
& \approx n\left(1-e^{-\frac{N}{n}}\right) .
\end{aligned}
$$

Even for moderate values of $n,(3)$ is very close to (2). For example, for $N=150$ and $n=1000$, the expected value of detected balls, i.e., sensors, would be $\simeq 139$.

To mitigate the effect of having several sensors set the same bit, we introduce a simple correction to the estimate (3). We observe that there should be roughly

$$
\frac{N}{n \cdot\left(1-e^{-N / n}\right)}
$$

sensors for each set bit in the bit vector. Therefore, if the number of set bits in the aggregated bit vector is $N^{\prime}$, then we can have the corrected estimate of $N$ as

$$
N_{\text {corr }}=\left\|N^{\prime} \cdot \frac{N^{\prime}}{n \cdot\left(1-e^{-N^{\prime} / n}\right)}\right\| .
$$

In our example of 150 balls and 1000 bins, after the correction, we will estimate 149 balls rather than the previous 139 balls. Figure 3 represents the improvement for two scenarios of different numbers of bins and balls.

The error percentage after the correction is shown in Figure 4 . With a wise selection of the number of bins, $n$, the error percentage can be made very small. For example, with 3000 bins and up to 200 balls, the error is zero. 


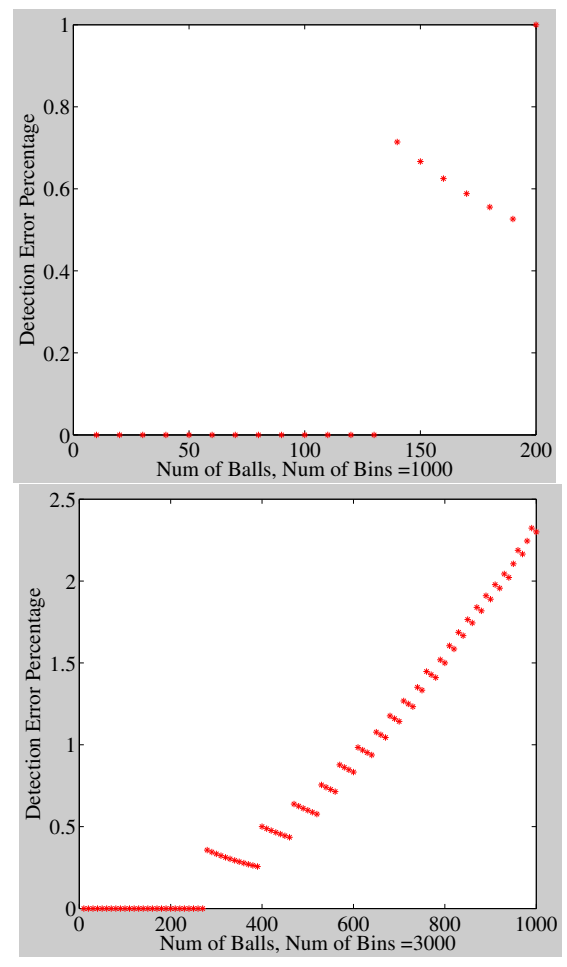

Fig. 4. The error percentage for probabilistic counting.

How should the FR select $n$ ? First, we note that

$$
\lim _{n \rightarrow \infty} n\left(1-e^{-\frac{N}{n}}\right)=N \text {. }
$$

In other words, to get the exact value of $N$, the length $n$ of the bit vector should be infinite. Naturally, this is infeasible. Assume that the FR has reasons to believe that $N$ is an upper bound on the number of sensors with the desired capability. Assume further that, for some $a,(0<a<1)$, the FR estimates that her mission needs $a \cdot N$ sensors with the given capability. In order to choose a value for $n$, the FR uses (3) by setting

$$
a N=n\left(1-e^{-\frac{N}{n}}\right)
$$

which is equivalent to

$$
1-e^{-\frac{N}{n}}=a \frac{N}{n} .
$$

Write $x=\frac{N}{n}$ and approximate the Taylor expansion of $1-e^{-x}$ as $\approx x-\frac{x^{2}}{2}+\frac{x^{3}}{6}$. Plugging all this back into (5) we obtain

$$
x-\frac{x^{2}}{2}+\frac{x^{3}}{6}=a x
$$

which, after division by $x \neq 0$ yields

$$
x^{2}-3 x+6(1-a)=0 .
$$

This latter quadratic equation can be solved for $x$. Before doing so, we note that in order to obtain a decent estimate of $N$, we must have $x=\frac{N}{n}<1$. We also note that (6) has a (real) root less than 1 if and only if $a \in\left[\frac{2}{3}, 1\right)$. Moreover, this root is

$$
x=\frac{3-\sqrt{9-24(1-a)}}{2} .
$$

This yields the desired value for $n$ as a function of $N$ and $a$

$$
n=\frac{2 N}{3-\sqrt{9-24(1-a)}}
$$

or, equivalently,

$$
n=\frac{N(3+\sqrt{9-24(1-a)})}{12(1-a)} .
$$

Finally, our probabilistic counting method can be extended to collect sensor capabilities at the same time by setting encoded value bytes in a byte vector rather than bits in a bit vector. For example, assume there are 8 types of sensors available in general. A node that has sensors of type 1, 3, and 5 will set the byte as 00010101 . After this, instead of OR-ing bits, another function, e.g. MAX, will be applied to the corresponding bytes. At the end, the aggregated byte vector will indicate the estimated number of sensor nodes with different categories of sensor capabilities.

\section{B. Extension to a scalable solution}

Operations running on sensor nodes can be separated into two categories: (I) ones that operate on a large number of sensor nodes (e.g., 100s of temperature sensors in various rooms of a building); (II) ones that operate on a limited number of nodes (e.g., air quality monitoring). In category I, i.e., high density, there is no need to know the exact number of sensor nodes. However, in category II, i.e., low density, the exact number of sensor nodes is required.

The method that we described in Section III-A performs very well for low density scenarios. Although the method retains a small detection error percentage as the number of nodes is increased, we are looking to extend the method for high density scenarios to achieve a lower error percentage. Furthermore, this extended solution will address the issues related to the existence of a non-ideal MAC and competition among nodes to access the wireless channel.

The extended scheme exploits the idea of spreading the capability collection (i.e., counting process) over space and time. However, this spreading could increase the length of the process. So, we try to optimize this spreading. First, spreading can be defined as follows:

- Time spreading: The capability collection of nodes in one sub-area should be spread over time, so there would be no collisions on the transmission of messages from collocated sensor nodes.

- $\quad$ Space spreading: We divide the area into subareas and collect the capability of each subarea in a separate inquiry. For this purpose, we can use the VI (described in Section II-B). Knowing the sectors of the virtual infrastructure, we can query each sector individually.

We use the VI to collect the sensor capabilities as follows. For simplicity, we assume that the number of sectors is equal to the number of coronas, i.e., the number of wedges is one. At time $t_{0}$, the inquirer asks the sensor nodes in the outermost corona to announce if they have the inquired sensor capability. Sensor nodes in that corona select a random number from a bit vector and set that bit to 1 if they have the inquired capability. 
TABLE I. A SAMPLE COMBINED BIT VECTOR RECEIVED BY A FR, REPRESENTING THE SENSOR NODES WITH THE INQUIRED CAPABILITY.

\begin{tabular}{c||ccccccc}
\multicolumn{1}{c||}{} & \multicolumn{7}{c}{ Bit Vector } \\
\hline Corona 1 & 0 & $\cdots$ & 0 & 1 & 0 & $\cdots$ & 0 \\
\hline Corona 2 & 0 & 0 & $\cdots$ & $\cdots$ & 0 & 1 & 0 \\
\hline$\vdots$ & \multicolumn{1}{c}{} \\
\hline Corona k & 0 & $\cdots$ & 0 & 1 & 0 & $\cdots$ & 0
\end{tabular}

Next, they transmit this bit vector in packets within a duration of $\Delta$, which we henceforth call the transmission window. To avoid collisions between concurrent transmissions, the sensor nodes will choose their transmission time uniformly within $(0, \Delta)$. The minimum value for $\Delta$ can be designed as follows.

$$
\Delta_{\min }=\frac{K \cdot l \cdot N}{B},
$$

where $K$ represents the number of packets sent by a node, $l$ is the length of a packet, and $B$ is the available bandwidth. The value $\Delta_{\min }$ is based on the assumption that all nodes will have enough time to transmit their packet sequentially with the assumption of no overlap.

Since it is assumed that sensor nodes have a shorter communication range than the inquirer, the sensor nodes in the next corona will receive those packets. Next at time $t_{0}+\Delta$, the inquirer asks the nodes in the next corona to announce their capabilities. Again, each node selects a random number from the bit vector and sets it to 1 if they have the capability. In addition, they will $O R$ every bit vector they have received from the previous corona and, after aggregating them, attach them to their own bit vector. For example, if a node has received two bit vectors that have set the bits 45 and 178, respectively, then it aggregates them into one bit vector that has set both bits 45 and 178 to 1 . The aggregator node also may, for example, set the $32 \mathrm{nd}$ bit of its own vector to 1 . The final message that this node will transmit will have these two bit vectors: one with bits 45 and 178 and the second one with bit 32. This aggregation decreases the chance of bit loss due to the imperfect wireless channel. After aggregation and attachment, they transmit the combined bit vector. This pattern is repeated for all coronas. When the nodes in the last corona, i.e. the innermost corona, transmit the combined bit vector, the inquirer will receive it. The inquirer will make the final aggregation and will know how many capable sensors in each corona exist (Table I).

\section{Methodology}

In order to test our theoretical predictions and the efficiency of our probabilistic counting method we ran a series of experiments using IRIS motes and a set of simulations in NS-2.

\section{A. IRIS mote experiments}

First, we must be able to detect the presence of a specific type of sensor on the IRIS mote. There is no programmable access to determine this information, so we infer the presence of specific sensors. A sensor's readable pin is abstracted into a component with a descriptive name, Tempc, for example. If we include TempC in a program, we know that when we read TempC, we are reading the pin that corresponds to a temperature sensor. Thus, we read the pin, then call for the power to that sensor, if present, to be shut off, then read the pin
TABLE II. PARAMETERS OF EXPERIMENTS

\begin{tabular}{l|l|l|l} 
Experiment & Topology & Hops & Coronas \\
\hline A & linear & 1 & 7 \\
B & star & 1 & 1 \\
C & star & 2 & 2
\end{tabular}

again. If there is significant difference between these two sets of readings, we declare that the sensor in question is present. This method correctly detects the presence or absence of the MTS310 sensorboard on the IRIS mote platform.

We ran our experiments on eight IRIS motes. Seven motes are deployed as nodes which reported their sensing capability and one mote is employed as the base, i.e., the FR in our VI, which controls the counting process and aggregates the received bit vectors of sensor nodes. Several topologies, where motes are distributed in various numbers of coronas, are considered to evaluate the performance of our probabilistic counting process. Table II illustrates the parameters for our various experiment scenarios. In all experiments, we are assuming the size of the bit vector is equal to 3000 .

Each experiment consists of about 300 trials of sending inquiries by the base and receiving responses from sensor nodes. At intervals of a few seconds, the base mote sends a signal packet which contains a number corresponding to a corona. If a node receives a signal packet containing its corona number, it begins to transmit its bit vector, waiting a random amount of time before sending any packet. In the event that the bit vector is too large to fit into a single packet, it is split into several packets. An index is sent along with the each packet, indicating which piece of the bit vector the packet contains. When the base receives a packet containing a piece of the bit vector, it aggregates the received piece with the corresponding section of its own bit vector by applying the OR operation to each pair of bits. When a node receives a bit vector piece packet from a corona farther from the base than its own, it similarly aggregates that piece with its own bit vector.

1) Experiment-A: In this experiment, the motes are arranged in a straight line with one meter separating each mote. The base mote is positioned at one end. This experiment models a scenario where the base is communicating in a singlehop fashion with coronas, and each corona contains only one node and there is only one wedge. This is nearly the simplest possible setup and there were not predicted to be significant deviations from the theoretical expected number of sensors counted, due to the simple nature of the network.

Each experiment resulted in a nearly perfect match with the theoretical expected number of sensors. The average percent error with respect to the theoretical expected value was $0.235 \%$. Less than 10 packets in nearly 100,000 were lost.

2) Experiment- $B$ : In this experiment, all seven nodes are considered to be in one corona. The value of transmission window $\Delta$ is varied between $50-4500 \mathrm{~ms}$. The nodes are arranged circularly around the base at similar, but slightly varying, distances. Placing all available nodes into one corona is perhaps the most complex scenario we can achieve with so few motes. It is expected that some collisions will occur during the transmission of bit vectors.

With a strict $50 \mathrm{~ms}$ window, $52.5 \%$ of packets arrived 


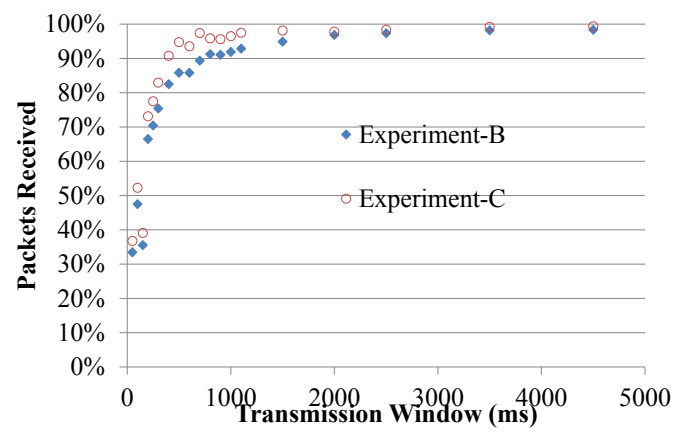

Fig. 5. Percentage of successful packet receptions

TABLE III. Simulation parameters

\begin{tabular}{|l|l|}
\hline Simulation time & $2500 \mathrm{~s}$ \\
\hline CSMA/CA MAC & $250 \mathrm{kbps}$ \\
\hline Communication range of inquirer & $700 \mathrm{~m}$ \\
\hline Communication range of sensor nodes & $30 \mathrm{~m}$ \\
\hline Transmission window $(\Delta)$ & $12,000 \mathrm{~ms}$ \\
\hline
\end{tabular}

late, resulting in a low average sensor count (1.11 out of 7). As shown in Figure 5, with a transmission window of 1500 ms, 93\% accuracy in packet reception was achieved. With a window of $4500 \mathrm{~ms}, 98 \%$ accuracy was achieved. The point at which the packet reception does not change significantly (approximately at $1500 \mathrm{~ms}$ ) corresponds to Equation (8) for our scenario parameters. For any transmission window size larger than $1500 \mathrm{~ms}$, more than 6.5 out of 7 motes are detected.

3) Experiment- $C$ : In this experiment, we form a twocorona topology. Four motes were located in the outer corona and three motes in the inner corona. The goal is to simulate a multihop scenario to be contrasted with Experiment-B. It was expected that this topology would increase the accuracy of the sensor counting process, as there would be fewer chances for collisions than the topology of Experiment-B for the same window transmission size, as fewer sensors would transmit simultaneously. As expected, with fewer motes per corona, more than 6.75 out of 7 sensors are detected with any transmission window size larger than $1500 \mathrm{~ms}$. This is due to a better packet reception percentage as illustrated in Figure 5.

\section{B. Simulation results}

We ran a number of simulation experiments in order to assess the accuracy of our probabilistic counting strategy, while taking into account the wireless channel condition, i.e., collisions and path loss, in a larger scale rather than our experiments with only eight IRIS motes. We simulated this process in NS 2.29 [13]. The sensors were distributed uniformly at random in a circular disk of radius $700 \mathrm{~m}$, centered at the FR. The number of sensors varies between 10 to 1000. Other simulation parameters are shown in Table III.

Figure 6 illustrates that the difference between the ideal wireless communication (i.e., theory) and real wireless communication (i.e., simulation) is insignificant for less than 300 nodes. This confirms that correction factor (4), time spreading, space spreading and repeating the previous bit vectors in multihop fashion compensate for the imperfection of the wireless channel and large number of sensor nodes. Again, notice that

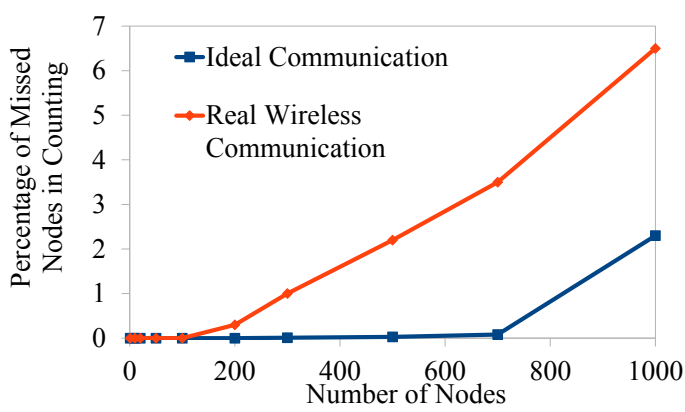

Fig. 6. Comparing the detection error percentage for theory and simulation.

for about 200 nodes, the error is almost zero. This means that as long as $n$ is wisely selected large enough (with the assumption of knowing the upper bound of $N$ ), the probabilistic counting method would have a high accuracy.

\section{CONCLUding REMARKS}

In this paper, we proposed a light-weight method for asset registry in emergency situations. Particularly, we designed a probabilistic counting protocol to discover the capabilities of independently deployed sensors in an area affected by a disaster. Although it is a probabilistic approach, theoretical and experimental results show that the error of the capability collection process would be almost zero with a smart design.

\section{REFERENCES}

[1] A. Boukerche, R. Araujo, and L. Villas, "A wireless actor and sensor networks QoS-Aware routing protocol for the emergency preparedness class of applications," in Proceedings of the IEEE Conference on Local Computer Networks, 2006, pp. 832-839.

[2] B. Manoj and A. H. Baker, "Communication challenges in emergency response," Commun. ACM, vol. 50, no. 3, pp. 51-53, Mar. 2007.

[3] L. Oliveira and J. Rodrigues, "Wireless sensor networks: A survey on environmental monitoring," J. Commun., vol. 6, no. 2, 2011.

[4] S. Olariu, S. Mohrehkesh, and M. C. Weigle, "Toward aggregating time-discounted information," in Proceedings of the ACM Workshop on Mission-oriented Wireless Sensor Networking, 2013, pp. 57-66.

[5] S. Olariu, S. Mohrehkesh, X. Wang, and M. C. Weigle, "On aggregating information in actor networks," SIGMOBILE Mob. Comput. Commun. Rev., vol. 18, no. 1, pp. 85-96, Feb. 2014.

[6] Y. Zhang, L. Ackerson, D. Duff, C. Eldershaw, and M. Yim, "Stam: a system of tracking and mapping in real environments," IEEE Wireless Communications, vol. 11, no. 6, pp. 87-96, Dec 2004.

[7] S. Olariu, M. Eltoweissy, and M. Younis, "ANSWER: AutoNomouS netWorked sEnsoR system," Journal of Parallel and Distributed Computing, vol. 67, no. 1, pp. 111-124, 2007.

[8] S. Olariu and $\mathrm{Q}$. $\mathrm{Xu}$, "A simple and robust virtual infrastructure for massively deployed wireless sensor networks," Computer Communications, vol. 28, no. 13, pp. 1505-1516, 2005.

[9] J. W. Hui and D. Culler, "The dynamic behavior of a data dissemination protocol for network programming at scale," in Proceedings of ACM SenSys, 2004, pp. 81-94.

[10] ITU, "Guidelines to select emergency number for public telecommunications networks," E.161.1, Sep. 2008.

[11] S. Olariu, A. Wadaa, L. Wilson, and M. Eltoweissy, "Wireless sensor networks: Leveraging the virtual infrastructure," Netwrk. Mag. of Global Internetwkg., vol. 18, no. 4, Jul. 2004.

[12] W. Feller, An Introduction to Probability Theory and Its Applications 2-nd Edition. John Wiley \& Sons, 1957, vol. 1.

[13] “ns2: The network simulator," http://nsnam.isi.edu/nsnam. 